\title{
A statistical selection of on-plate sites based on a VLBI global solution
}

\author{
Jinling Li, Bo Zhang, Ming Zhao, and Guangli Wang \\ Shanghai Astronomical Observatory, National Astronomical Observatories, Chinese Academy of Sciences, Shanghai 200030, P. R. China
}

(Received June 7, 2001; Revised August 23, 2001; Accepted September 12, 2001)

\begin{abstract}
In the compilation of the conventional terrestrial reference frame with modern space geodetic techniques, one of the important issues is to select those on-plate sites. Considering that whether the motion of a site can be modeled or not by plate rigid motion is not necessarily dependant on the distance from the site to the plate boundary or deforming zones, we propose a statistical selection of on-plate sites. By applying this selection method on a VLBI global solution, the followings are shown. (1) The statistically selected on-plate sites are more than and in consistency globally with the VLBI primary sites of the International Terrestrial Reference Frame 2000 (ITRF2000). (2) The statistical selection emphasizes the consistency between the site motion and the plate rigid motion but ignores the distance from the site to the plate boundaries or deforming zones. (3) Some of the statistically selected on-plate sites are not primaries in ITRF2000. However, by including these sites the estimations of plate rigid motion are not changed significantly. Instead, the precision of estimated parameters is increased due to the improvement in the site geometric distribution. (4) Details of our analysis show that RICHMOND can be taken as a on-plate site, but it is not in the list of those most firmly on-plate. This site is usually used as one of the velocity constraint sites in VLBI global analysis. We recommend using ALGOPARK, FD-VLBA or NRAO_140 as substitutes.
\end{abstract}

\section{Introduction}

For an ideal terrestrial reference frame, its motion relative to the inertial reference frame represents the motion of the Earth as a whole, while the motion of the Earth relative to this terrestrial frame is only local motion rather than any net translation or net rotation. Such an ideal terrestrial reference frame is usually defined by the Tisserand condition (Boucher, 1989):

$$
\begin{aligned}
& \int \vec{v} d m=0 \\
& \int \vec{r} \times \vec{v} d m=0
\end{aligned}
$$

where $\vec{r}$ and $\vec{v}$ is respectively the position and the velocity of a mass point $d m$ of the Earth, and the domain of integration is the whole Earth. Due to the inaccessibility of the motion of the Earth's interior, the domain of integration is conventionally simplified to be the Crust.

If there are evenly and densely distributed sites on the Crust with precise determinations of positions and velocities, a terrestrial frame without net translation and rotation relative to the Crust can be defined by satisfying the discrete Tisserand condition:

$$
\begin{aligned}
& \sum \vec{v} d m=0 \\
& \sum \vec{r} \times \vec{v} d m=0 .
\end{aligned}
$$

If the Crust is taken as being composed of rigid plates and the relative motions among plates are known by some

Copy right (c) The Society of Geomagnetism and Earth, Planetary and Space Sciences (SGEPSS); The Seismological Society of Japan; The Volcanological Society of Japan; The Geodetic Society of Japan; The Japanese Society for Planetary Sciences. means, then by applying the constraint of no net translation and rotation, an absolute plate motion model can be deduced to realize a conventional terrestrial reference frame, which is "fixed" to the Crust.

By using the earthquake slip vectors and magnetic anomalies averaged over about $3 \mathrm{Myr}$, the relative motion parameters among plates are determined (DeMets et al., 1994). However this determination may differ slightly (and sometimes significantly) from the modern space geodetic measurements (MacMillan and Ma, 1999; Kogan et al., 2000). If only the modern space geodetic measurements are used, because of the existence of local deformations and the uneven and sparse distribution of sites, neither the relative motion parameters of all the plates nor the ideal terrestrial reference frame satisfying the discrete Tisserand condition can be obtained. Accordingly, it is conventional to realize the terrestrial reference frame by combining the modern space geodetic measurements and the plate motion model from paleo-geophysical data. In the realization of the latest version of the International Terrestrial Reference Frame (ITRF2000) (cf. http://lareg.ensg.ign.fr/ ITRF / ITRF $2000-\mathrm{PA}$ /datum.html), for instance, the coordinates of a set of selected sites (primaries) at a specified epoch are used to define the orientation of ITRF2000, while the orientation evolution is defined by applying no net rotation to the velocities of primaries relative to NNRNuvella (DeMets et al., 1994). Therefore, the selected sites are important because they are primaries to define the orientation and to maintain the time evolution of the conventional terrestrial reference frame.

Due to the importance of primaries in the conventional terrestrial frame, they should be of some basic charac- 
teristics. For instance, (1) their positions and velocities are precisely determined with modern space geodetic techniques; (2) their motions are in good consistency with the plate rigid motion and (3) they should be distributed with reasonable geometry and density. Heki (1996) selected permanent VLBI stations located in areas more than 500 $\mathrm{km}$ from the nearest plate boundary to define the kinematical reference frame. In ITRF2000, the criteria for selection of primaries are (1) continuously observed during at least 3 years; (2) located far away from plate boundaries and deforming zones; (3) velocity accuracy (as result of ITRF2000) better than $3 \mathrm{~mm} / \mathrm{y}$ and (4) velocity residuals less than $3 \mathrm{~mm} / \mathrm{y}$ for at least 3 different solutions (cf. http://lareg.ensg.ign.fr/ITRF/ ITRF2 $000-\mathrm{PA}$ /datum.html). However, considering that whether the motion of a site can be modeled or not by plate rigid motion is not necessarily dependant on the distance from the site to the plate boundary or deforming zones, and that, since the number of sites of modern space techniques are limited, all the sites with motions consistent with the plate rigid motion are essential to the determination of contemporary plate motion and to the realization of the terrestrial reference frame, hereby we propose a statistical selection of the on-plate sites.

\section{A Statistical Selection of On-Plate Sites}

Let $\vec{v}_{j i}$ be the space geodetic measurement of the velocity of the $i$ th station on the $j$ th plate and be expressed as,

$$
\vec{v}_{j i}=\vec{v}_{j i}^{0}+\vec{v}_{j i}^{l}+\vec{\epsilon}_{j i}
$$

where $\vec{v}_{j i}^{0}, \vec{v}_{j i}^{l}$ and $\vec{\epsilon}_{j i}$ represent respectively the plate rigid motion, the local deformation and the observation noise at the $i$ th station. $\quad \vec{v}_{j i}^{0}$ may differ from $\vec{v}_{j i}^{m}$ which represents the prediction of plate motion model. Let this difference be modeled by a set of systematic parameters $\vec{T}_{j}$ and $Q_{j}$ of the $j$ th plate as the following,

$$
\vec{v}_{j i}^{0}=\vec{v}_{j i}^{m}+\vec{T}_{j}+Q_{j} \vec{r}_{j i}
$$

where $\vec{r}_{j i}$ represents the geocentric position of the $i$ th station; $\vec{T}_{j}$ represents the translation rate,

$$
\vec{T}_{j}=\left[T_{x}, T_{y}, T_{z}\right]_{j}^{T}
$$

the superscript $T$ represents the transverse of matrix; in the first order approximation,

$$
Q_{j}=\left[\begin{array}{ccc}
D & R_{z} & -R_{y} \\
-R_{z} & D & R_{x} \\
R_{y} & -R_{x} & D
\end{array}\right]_{j}
$$

where $R_{x}, R_{y}$ and $R_{z}$ are the rates of small angular rotations around the corresponding coordinate axes, $D$ is the rate of the scale factor. Finally Eq. (3) can be expressed as

$$
\vec{v}_{j i}=\vec{v}_{j i}^{m}+\vec{T}_{j}+Q_{j} \vec{r}_{j i}+\vec{v}_{j i}^{l}+\vec{\epsilon}_{j i}
$$

where $\vec{v}_{j i}^{m}$ can be the prediction of the plate motion model or the a priori value of the station motion corresponding to the plate rigid motion. Hereafter we will refer it simply as the prediction of plate motion model. In the geocentric form Eq. (7) is as

$$
\begin{aligned}
{\left[\begin{array}{l}
v_{x} \\
v_{y} \\
v_{z}
\end{array}\right]_{j i}=\left[\begin{array}{l}
v_{x} \\
v_{y} \\
v_{z}
\end{array}\right]_{j i}^{m}+\left[\begin{array}{l}
T_{x} \\
T_{y} \\
T_{z}
\end{array}\right]_{j} } \\
+\left[\begin{array}{ccc}
D & R_{z} & -R_{y} \\
-R_{z} & D & R_{x} \\
R_{y} & -R_{x} & D
\end{array}\right]_{j}\left[\begin{array}{l}
x \\
y \\
z
\end{array}\right]_{j i} \\
+\left[\begin{array}{c}
v_{x} \\
v_{y} \\
v_{z}
\end{array}\right]_{j i}^{l}+\left[\begin{array}{c}
\epsilon_{x} \\
\epsilon_{y} \\
\epsilon_{z}
\end{array}\right]_{j i} .
\end{aligned}
$$

In Eq. (7), $\vec{v}_{j i}$ and $\vec{v}_{j i}^{m}$ are known quantities; $\vec{T}_{j}$ and $Q_{j}$ are unknowns; with adequate observations $\vec{\epsilon}_{j i}$ will obey the normal distribution; $\vec{v}_{j i}^{l}$ corresponds to the local deformation. If we solve for the systematic parameters through Eq. (7) by a weighted least squares adjustment to all the stations on the $j$ th plate with the ignorance of $\vec{v}_{j i}^{l}$ and let $\vec{v}_{j i}^{l \prime}$ be the post-fit residual, then $\vec{v}_{j i}^{l \prime}$ will be the combination of the observation noise and the part in $\vec{v}_{j i}^{l}$ which cannot be modeled by $\vec{T}_{j}$ and $Q_{j}$. It is accordingly reasonable that for a large absolute value of $\vec{v}_{j i}^{l}$ the corresponding absolute value of $\vec{v}_{j i}^{l \prime}$ will be large with a high possibility. With this consideration, we take $\vec{v}_{j i}^{l \prime}(i=1, \ldots, n ; n$ is the number of stations on the $j$ th plate) as a random series and calculate $\sigma$, the variance of this series. We kick out those stations with absolute values of $\vec{v}_{j i}^{l \prime}$ larger than $f \sigma$ ( $f$ is a scaling factor and will be discussed in the following paragraph). We re-estimate the parameters $\vec{T}_{j}$ and $Q_{j}$ by using only those kept stations. Statistically speaking, because those stations within large deforming zones (with high possibility) are not included, the new estimation will be more close to the systematic difference between the observations and the model predictions in comparison with the first time estimation. We repeat the above estimating and kicking-out calculation until the point at which though there are stations being statistically kicked out, the estimation to systematic parameters and the variance of post-fit residuals are not changed significantly before and after the kicking-out operation. The motions of the finally kept stations should be in good consistency with the rigid motion of the plate. The statistically selected stations can be taken as candidates of on-plate sites.

In order to choose an appropriate value for $f$, let us classify all the stations on the $j$ th plate into two groups. In Group 1 suppose only on-plate stations are included, the corresponding variance of the post-fit residual series and the sum of weightings are $\sigma_{1}$ and $P_{1}$ respectively. In Group 2 only stations within deforming zones are included, the corresponding quantities are $\sigma_{2}$ and $P_{2}$. Then the variance $(\sigma)$ of the post-fit residual series for all the stations on the $j$ th plate can be expressed as

$$
\sigma^{2}=\left(P_{1} \sigma_{1}^{2}+P_{2} \sigma_{2}^{2}\right) /\left(P_{1}+P_{2}\right) .
$$

If the systematic parameters in Eq. (7) are properly estimated, then $\sigma_{1}$ will be mainly corresponding to the observation noises, while $\sigma_{2}$ will be the combination of noises 
Table 1. The estimating and kicking-out process for NOAM.

\begin{tabular}{lrrrrr}
\hline Circle & 1 & 2 & 3 & 4 & 5 \\
Points for estimating & 44 & 23 & 17 & 16 & 14 \\
$T_{x}(\mathrm{~mm} / \mathrm{yr})$ & $-3.279 \pm 0.317$ & $-0.367 \pm 0.565$ & $-0.128 \pm 0.752$ & $-0.195 \pm 1.100$ & $-0.132 \pm 1.124$ \\
$T_{y}(\mathrm{~mm} / \mathrm{yr})$ & $-2.900 \pm 0.266$ & $-1.953 \pm 0.650$ & $-2.123 \pm 0.969$ & $-1.398 \pm 1.826$ & $-1.532 \pm 1.858$ \\
$T_{z}(\mathrm{~mm} / \mathrm{yr})$ & $-0.895 \pm 0.280$ & $0.509 \pm 0.705$ & $1.266 \pm 1.186$ & $1.671 \pm 2.207$ & $1.602 \pm 2.254$ \\
$D\left(10^{-8} / \mathrm{yr}\right)$ & $-0.026 \pm 0.003$ & $-0.028 \pm 0.007$ & $-0.038 \pm 0.008$ & $-0.033 \pm 0.008$ & $-0.034 \pm 0.008$ \\
$R_{x}(\mathrm{mas} / \mathrm{yr})$ & $0.033 \pm 0.011$ & $-0.003 \pm 0.028$ & $-0.020 \pm 0.048$ & $-0.046 \pm 0.093$ & $-0.040 \pm 0.095$ \\
$R_{y}(\mathrm{mas} / \mathrm{yr})$ & $0.080 \pm 0.013$ & $0.089 \pm 0.017$ & $0.089 \pm 0.021$ & $0.109 \pm 0.027$ & $0.107 \pm 0.027$ \\
$R_{z}(\mathrm{mas} / \mathrm{yr})$ & $-0.252 \pm 0.007$ & $-0.107 \pm 0.017$ & $-0.098 \pm 0.023$ & $-0.125 \pm 0.032$ & $-0.122 \pm 0.032$ \\
$\sigma(\mathrm{mas} / \mathrm{yr})$ & 3.35 & 1.12 & 1.03 & 0.89 & 0.89 \\
Points kicked-out & 21 & 6 & 1 & 2 & 0 \\
\hline
\end{tabular}

and local deformations. Accordingly, $\sigma_{2}$ will be larger than $\sigma_{1}$. Suppose $\sigma_{2}^{2}=\sigma_{1}^{2}+o^{2}$ and $o^{2}>0$, then from Eq. (9) the following can be obtained:

$$
\begin{aligned}
\sigma^{2} & =\left[\sigma_{1}^{2}\left(P_{1}+P_{2}\right)+P_{2} o^{2}\right] /\left(P_{1}+P_{2}\right) \\
& =\sigma_{1}^{2}+P_{2} o^{2} /\left(P_{1}+P_{2}\right)>\sigma_{1}^{2} \\
\sigma^{2} & =\left[\sigma_{2}^{2}\left(P_{1}+P_{2}\right)-P_{1} o^{2}\right] /\left(P_{1}+P_{2}\right) \\
& =\sigma_{2}^{2}-P_{1} o^{2} /\left(P_{1}+P_{2}\right)<\sigma_{2}^{2} .
\end{aligned}
$$

In other words, the variance of the post-fit residual series of all the stations is larger than that of the on-plate stations and is less than that of stations within deforming zones. So we can statistically select those on-plate sites by taking $1 \sigma$ as the criterion, i.e., $f=1$.

\section{A Statistical Selection of On-Plate Sites Based on a VLBI Global Solution}

VLBI is the space geodetic technique to provide the whole set of Earth Orientation Parameters (polar motion, length of day and celestial pole offsets). The radio celestial reference frame realized by VLBI is so far the nearest quasi-inertial reference frame. Due to the outstandingly high stability and precision in the observation of very long baseline, VLBI has been one of the principal techniques to realize the conventional terrestrial reference frame. The astrometric and geodetic VLBI has presently accumulated about 20 years of observations. The observations between August 1979 and December 1998 were analyzed by the geodetic VLBI group of Shanghai Astronomical Observatory (Li and Wang, 2000). The resultant global solution of the terrestrial reference frame (hereafter referred as SHATRF) was adopted as one of the three VLBI solutions of ITRF2000 (cf. http: / / lareg.ensg. ign.fr/ITRF/ ITRF2000/submissions.html).

In SHATRF there are 127 stations with geocentric coordinates and velocities. Some of the stations are mobile ones or with insufficient data. Their velocities are tied to the nearby permanent stations or the prediction of NNR-Nuvella in the VLBI global analysis. Obviously, these stations cannot be taken as on-plate sites and so they are excluded from the following analysis.
The Euler motion of a plate is modeled as the motion of plate on a sphere surface, which is corresponding to the horizontal motion of a site on the Crust. Accordingly, in the selection of on-plate sites by using Eq. (7) with a weighted least squares adjustment, the up component of station motion is not considered. The a priori value of the station motion in accordance with the plate rigid motion (i.e., the value of $\vec{v}_{j i}^{m}$ ) is taken as the prediction of NNR-Nuvella. The calculations are weighted based on formal uncertainties of observations. Residuals are tested by the $\chi^{2}$-test (A small additive variance is applied to compute the "true" standard errors and therefore to bring the reduced $\chi^{2}$ close to unity). Stations with absolute values of residuals larger than $\sigma$ (the variance of the residual series) are kicked out and the systematic parameters are estimated again by using only data of kept stations. The calculation is continued until the point where the variance of the residual series and the estimations of parameters are not changed significantly before and after one circle of calculation.

\subsection{North America plate}

SHATRF provides coordinates of 67 VLBI measuring points on the North America plate (NOAM). Among these points 44 are precisely determined with velocities. Table 1 shows the estimating and kicking-out process. In Circle 1, $\sigma$ is $3.35(\mathrm{~mm} / \mathrm{yr})$ and 21 points are kicked out. In Circle 2, $\sigma$ is dramatically decreased to 1.12 and six points are kicked out. In Circle 3, $\sigma$ is further decreased but only one point is kicked out. Till Circle 4 and 5, though there exist points being statistically kicked out, the value of $\sigma$ and the estimation to parameters are not changed significantly.

Table 2 shows the 14 points kept in Circle 5, the two points (NL-VLBA and FLAGSTAF) kicked out in Circle 4 and the point (RICHMOND) kicked out in Circle 3. About the 14 kept points in Circle 5, the absolute values of residuals for all the three components of velocity are less than $1.0 \mathrm{~mm} / \mathrm{yr}$. The velocities of most of the points are precisely determined with YUMA relatively weak. Our tests show that whether YUMA is included or not the estimations of systematic parameters are not changed significantly, which means that the motion of YUMA is in good consistency with the plate rigid motion of NOAM. This consistency may be changed as the possible accumulation of observations and a 
Table 2. The statistically selected on-plate sites on NOAM.

\begin{tabular}{|c|c|c|c|c|c|c|c|c|c|}
\hline \multirow[b]{2}{*}{ No } & \multirow[b]{2}{*}{ Sites } & \multirow{2}{*}{$\begin{array}{l}\text { Long. } \\
\text { (deg.) }\end{array}$} & \multirow{2}{*}{$\begin{array}{c}\text { Lat. } \\
\text { (deg.) }\end{array}$} & \multicolumn{3}{|c|}{ Uncertainty $(\mathrm{mm} / \mathrm{yr})$} & \multicolumn{3}{|c|}{ Residual (mm/yr) } \\
\hline & & & & $v_{x}$ & $v_{y}$ & $v_{z}$ & $v_{x}$ & $v_{y}$ & $v_{z}$ \\
\hline 1 & WESTFORD & -71.49 & 42.61 & 0.064 & 0.078 & 0.079 & 0.186 & 0.013 & 0.023 \\
\hline 2 & HAYSTACK & -71.49 & 42.62 & 0.178 & 0.285 & 0.296 & -0.507 & -0.463 & -0.228 \\
\hline 3 & HN-VLBA & -71.99 & 42.93 & 0.377 & 0.645 & 0.670 & -0.707 & 0.326 & 0.632 \\
\hline 4 & GORF7120 & -76.83 & 39.02 & 0.479 & 0.955 & 0.968 & 0.025 & 0.669 & 0.846 \\
\hline 5 & MARPOINT & -77.23 & 38.37 & 0.648 & 1.361 & 1.377 & -0.212 & -0.459 & -0.454 \\
\hline 6 & NRAO20 & -79.83 & 38.44 & 0.147 & 0.231 & 0.234 & 0.236 & -0.550 & -0.713 \\
\hline 7 & NRAO_140 & -79.84 & 38.44 & 0.148 & 0.349 & 0.352 & 0.022 & -0.045 & -0.040 \\
\hline 8 & NRAO85 3 & -79.84 & 38.43 & 0.144 & 0.271 & 0.273 & -0.208 & -0.168 & -0.142 \\
\hline 9 & ALGOPARK & -78.07 & 45.96 & 0.087 & 0.142 & 0.144 & 0.376 & 0.777 & 0.633 \\
\hline 10 & FD-VLBA & -103.94 & 30.64 & 0.177 & 0.297 & 0.291 & -0.472 & -0.298 & -0.642 \\
\hline 11 & LA-VLBA & -106.25 & 35.78 & 0.153 & 0.235 & 0.241 & -0.112 & -0.258 & -0.447 \\
\hline 12 & PIETOWN & -108.12 & 34.30 & 0.124 & 0.189 & 0.197 & 0.471 & 0.376 & 0.712 \\
\hline 13 & KP-VLBA & -111.61 & 31.96 & 0.354 & 0.470 & 0.476 & 0.744 & -0.130 & 0.289 \\
\hline 14 & YUMA & -114.20 & 32.94 & 3.237 & 4.114 & 4.117 & 0.025 & 0.144 & 0.259 \\
\hline 15 & NL-VLBA & -91.57 & 41.77 & 0.211 & 0.576 & 0.575 & -0.693 & -0.711 & -0.923 \\
\hline 16 & FLAGSTAF & -111.63 & 35.21 & 2.435 & 3.435 & 3.514 & 0.532 & 0.615 & 1.063 \\
\hline 17 & RICHMOND & -80.38 & 25.61 & 0.093 & 0.251 & 0.207 & -1.459 & -0.450 & -0.127 \\
\hline
\end{tabular}

precise determination of the velocity, which requires further tests in the future.

Concerning the two points being kicked out in Circle 4 , the velocity of FLAGSTAF is not very precise, but the motion is in good consistency with the plate rigid motion, which is similar to the case of YUMA as mentioned above. NL-VLBA is a primary site of ITRF2000; its motion is precisely determined. The two points are kicked out because of a relatively large absolute value of residual in the $z$ component of motion. However, as shown in Table 1, the estimations of parameters are almost the same in Circle 3 and 4 , it is reasonable to take the two points as on-plate.

The case for RICHMOND is unanticipated. Its motion is very precisely determined. In VLBI global analysis RICHMOND is usually used as a station for the terrestrial frame evolution constraint along with WESTFORD on NOAM. However, RICHMOND is kicked out in Circle 3, which leads to a decrease in $\sigma$ by more than $10 \%$. As shown in Table 1, the corresponding estimations of parameters in the first two circles are different tremendously and in the last two circles are only slightly. Circle 3 is in the middle of the calculation and the estimations of parameters are close to those in Circle 4. Accordingly, it is reasonable to take RICHMOND as on-plate. However, the difference between its motion and the statistically determined plate rigid motion is small and detectable. As shown in Table 2, RICHMOND is kicked out because of a large absolute value of residual in the $x$-component. It is worthwhile to notice that the motions of ALGOPARK, FD-VLBA and NRAO_140 are as precisely determined by VLBI as RICHMOND but with rather small residuals. It is recommended to take ALGOPARK, FD-VLBA or NRAO_140 as one of the evolution constraint stations in VLBI global analysis instead of RICH-
MOND.

Figure 1 shows the geometric distribution of the statistically selected on-plate stations (filled circles) and kicked out stations (hollow circles) on NOAM. The VLBI primaries (hollow squares) in ITRF2000 are also shown for comparison. The magnitude and direction of the horizontal residuals are indicated by the length and direction of the short lines. The scale is shown at the lower left corner of the figure. From this figure it is clear that 12 among the 17 statistically selected stations are the primaries or very near geometrically to the primaries in ITRF2000. The rest five stations are LAVLBA, PIETOWN, FLAGSTAF, KP-VLBA and YUMA, which are in the west of FD-VLBA. The velocity precision of FLAGSTAF and YUMA is lower than $3 \mathrm{~mm} / \mathrm{yr}$ in SHATRF and so the two stations are not primaries of ITRF2000. The other three stations are of high velocity precision but are very near to the boundary of NOAM and so are not primaries of ITRF2000 either. However, whether the five stations are included or not, the estimations to parameters are not changed significantly and so they are taken as on-plate in the statistical selection.

\subsection{Eurasia plate}

On the Eurasia plate (EURA), there are 31 VLBI stations, among which 12 are with sufficient observations to determine the velocity. Due to the small number of stations the estimation of parameters are not so stable as for NOAM. The estimating and kicking-out calculation for EURA is shown in Table 3, from which it is clear that though there are stations (YEBES and EFLSBERG) being statistically kicked out in Circles 3 and 4, the variance of residual series and the estimations of parameters are not changed significantly. Table 4 lists out the kept stations in Circle 5 and those kicked out in Circles 3 and 4. From this table it is 


\section{Relative motions of $V L B \mid$ stations on NOAM}

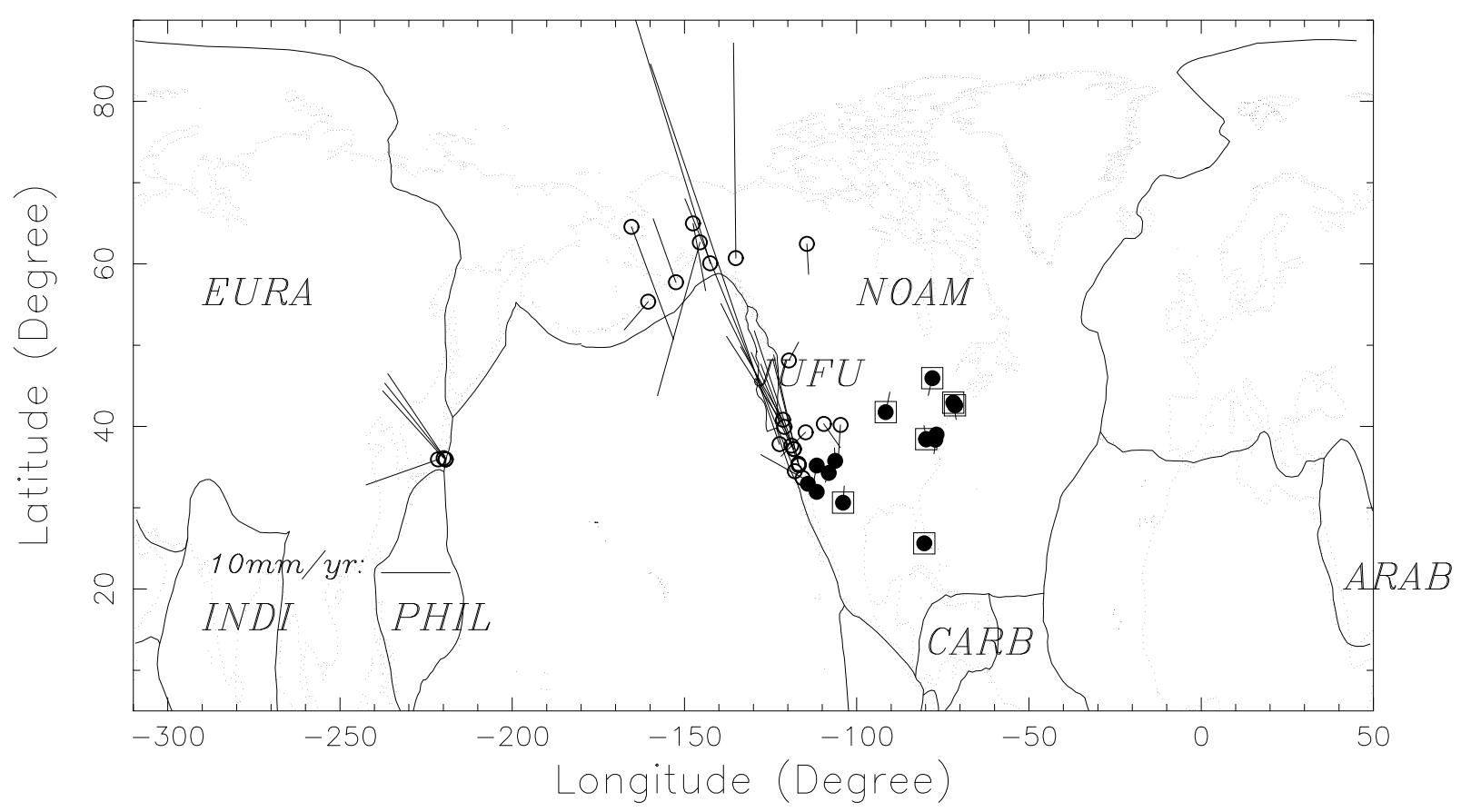

Fig. 1. The distribution of VLBI stations on NOAM. Filled circles, hollow circles and hollow squares represent respectively the statistically selected on-plate stations, the kicked out stations and the VLBI primaries in ITRF2000.

\section{Relative motions of VLBI stations on EURA}

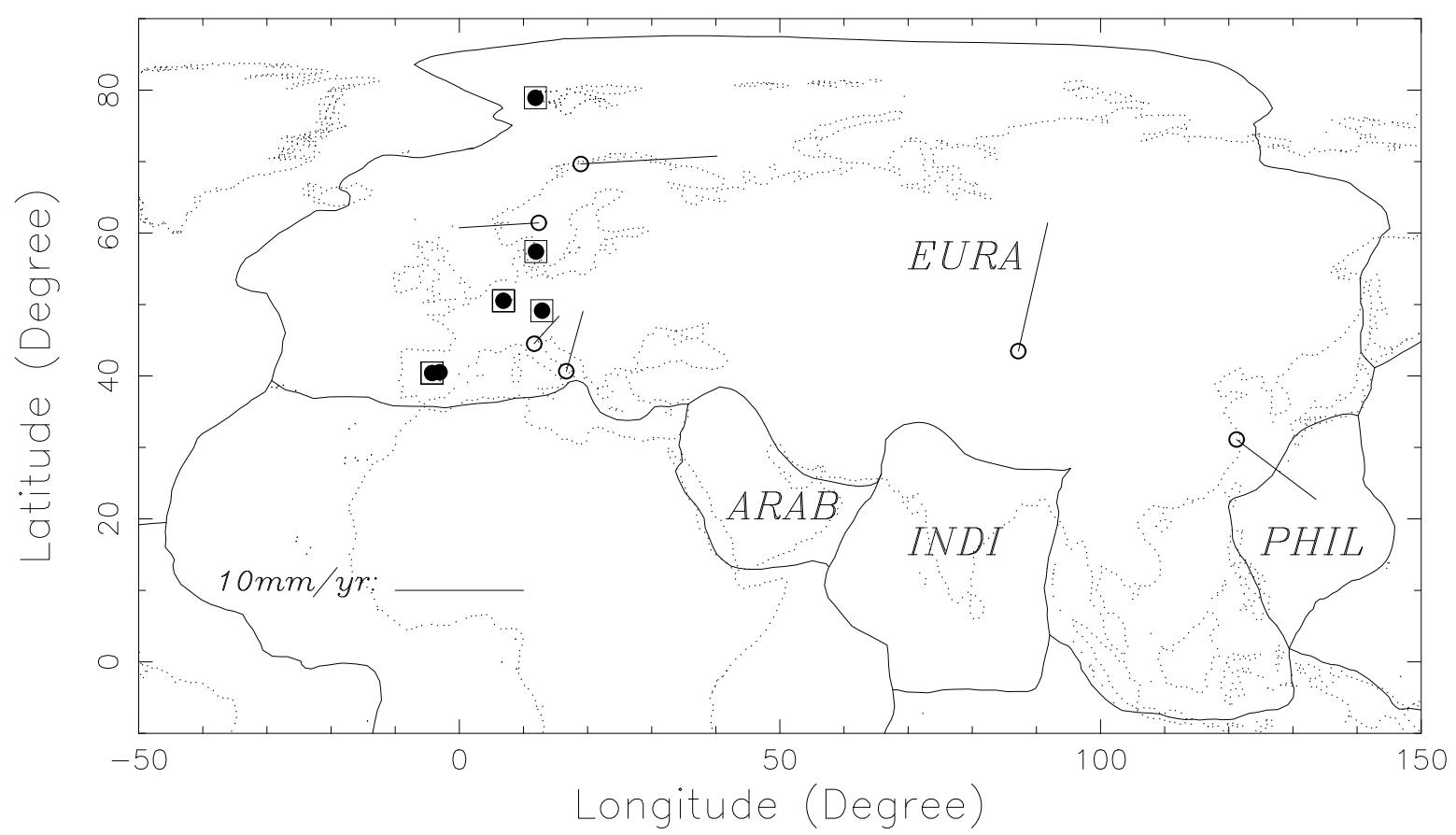

Fig. 2. The distribution of VLBI stations on EURA (Symbols mean the same as in Fig. 1).

shown that YEBES and EFLSBREG are relatively large in the absolute value of the $z$ - and $y$-component residual respectively. The velocity of all the six stations in Table 4 is very precisely determined with YEBES relatively weak.
The distribution of VLBI stations on EURA and the horizontal velocity residuals are shown in Fig. 2, in which the symbols mean the same as in Fig. 1. From Fig. 2 it is clear that the statistically selected sites are in good consistency 
Table 3. The estimating and kicking-out calculation for EURA.

\begin{tabular}{lrrrrr}
\hline Circle & 1 & 2 & 3 & 4 & 5 \\
Stations for fitting & 12 & 7 & 6 & 5 & 4 \\
$T_{x}(\mathrm{~mm} / \mathrm{yr})$ & $0.523 \pm 0.583$ & $-0.595 \pm 1.094$ & $-0.529 \pm 1.143$ & $-0.516 \pm 1.160$ & $-0.538 \pm 1.179$ \\
$T_{y}(\mathrm{~mm} / \mathrm{yr})$ & $3.429 \pm 0.780$ & $-0.294 \pm 2.990$ & $1.290 \pm 3.123$ & $0.753 \pm 3.146$ & $0.903 \pm 3.228$ \\
$T_{z}(\mathrm{~mm} / \mathrm{yr})$ & $0.461 \pm 0.572$ & $-1.488 \pm 0.911$ & $-1.107 \pm 0.977$ & $-1.051 \pm 0.992$ & $-1.074 \pm 1.000$ \\
$D\left(10^{-8} / \mathrm{yr}\right)$ & $-0.018 \pm 0.007$ & $0.024 \pm 0.014$ & $0.017 \pm 0.015$ & $0.017 \pm 0.015$ & $0.017 \pm 0.015$ \\
$R_{x}(\mathrm{mas} / \mathrm{yr})$ & $-0.020 \pm 0.030$ & $0.061 \pm 0.088$ & $0.005 \pm 0.092$ & $0.020 \pm 0.093$ & $0.015 \pm 0.095$ \\
$R_{y}(\mathrm{mas} / \mathrm{yr})$ & $-0.008 \pm 0.021$ & $0.021 \pm 0.035$ & $0.022 \pm 0.037$ & $0.021 \pm 0.037$ & $0.021 \pm 0.038$ \\
$R_{z}(\mathrm{mas} / \mathrm{yr})$ & $0.162 \pm 0.015$ & $0.076 \pm 0.054$ & $0.073 \pm 0.056$ & $0.057 \pm 0.057$ & $0.058 \pm 0.058$ \\
$\sigma(\mathrm{mas} / \mathrm{yr})$ & 2.23 & 1.25 & 0.61 & 0.49 & 0.53 \\
Kicked out stations & 5 & 1 & 1 & 1 & 0 \\
\hline
\end{tabular}

Table 4. The kept stations and their residuals for EURA.

\begin{tabular}{|c|c|c|c|c|c|c|c|c|c|}
\hline \multirow[b]{2}{*}{ No } & \multirow[b]{2}{*}{ Sites } & \multirow{2}{*}{$\begin{array}{l}\text { Long. } \\
\text { (deg.) }\end{array}$} & \multirow{2}{*}{$\begin{array}{l}\text { Lat. } \\
\text { (deg.) }\end{array}$} & \multicolumn{3}{|c|}{ Uncertainty (mm/yr) } & \multicolumn{3}{|c|}{ Residual (mm/yr) } \\
\hline & & & & $v_{x}$ & $v_{y}$ & $v_{z}$ & $v_{x}$ & $v_{y}$ & $v_{z}$ \\
\hline 1 & DSS65 & -4.25 & 40.43 & 0.384 & 0.126 & 0.386 & 0.325 & 0.430 & -0.119 \\
\hline 2 & NYALES20 & 11.87 & 78.93 & 0.147 & 0.113 & 0.291 & 0.034 & 0.043 & 0.050 \\
\hline 3 & ONSALA60 & 11.93 & 57.40 & 0.120 & 0.059 & 0.128 & -0.345 & 0.349 & 0.184 \\
\hline 4 & WETTZELL & 12.88 & 49.15 & 0.087 & 0.033 & 0.090 & 0.116 & -0.191 & -0.149 \\
\hline 5 & YEBES & -3.09 & 40.52 & 1.927 & 0.598 & 1.924 & -0.321 & -0.021 & 0.569 \\
\hline 6 & EFLSBERG & 6.88 & 50.52 & 0.353 & 0.140 & 0.359 & 0.164 & -0.696 & -0.014 \\
\hline
\end{tabular}

Table 5. The selection process on PCFC.

\begin{tabular}{lrrr}
\hline Circle & 1 & 2 & 3 \\
Stations for fitting & 13 & 4 & 5 \\
$T_{x}(\mathrm{~mm} / \mathrm{yr})$ & $-1.585 \pm 1.021$ & $-2.928 \pm 1.527$ & $-2.904 \pm 1.509$ \\
$T_{y}(\mathrm{~mm} / \mathrm{yr})$ & $2.245 \pm 2.344$ & $-4.332 \pm 4.879$ & $-4.339 \pm 4.862$ \\
$T_{z}(\mathrm{~mm} / \mathrm{yr})$ & $8.834 \pm 3.396$ & $-1.886 \pm 7.021$ & $-1.930 \pm 6.973$ \\
$D\left(10^{-8} / \mathrm{yr}\right)$ & $-0.060 \pm 0.012$ & $-0.050 \pm 0.015$ & $-0.049 \pm 0.014$ \\
$R_{x}(\mathrm{mas} / \mathrm{yr})$ & $-0.038 \pm 0.099$ & $0.193 \pm 0.212$ & $0.193 \pm 0.211$ \\
$R_{y}(\mathrm{mas} / \mathrm{yr})$ & $0.315 \pm 0.092$ & $0.013 \pm 0.183$ & $0.012 \pm 0.181$ \\
$R_{z}(\mathrm{mas} / \mathrm{yr})$ & $-0.358 \pm 0.050$ & $-0.204 \pm 0.094$ & $-0.203 \pm 0.094$ \\
$\sigma(\mathrm{mas} / \mathrm{yr})$ & 1.59 & 1.23 & 1.09 \\
Kicked out stations & 9 & -1 & 0 \\
\hline
\end{tabular}

with the VLBI primaries of ITRF2000.

\subsection{Pacific plate}

About the Pacific plate (PCFC), among the 16 VLBI stations 13 are determined with velocities. The statistical selection process is shown in Table 5. There are three circles of calculation and the number of kicked-out stations in Circle 2 is -1 . In the calculation, the stations used to estimate the parameters of Circle $i$ are those kept in Circle $i-1$. After the new estimation in Circle $i$ the variance $(\sigma)$ of residual series is calculated. Then all the residuals (including those re-evaluated ones with the newly estimated parameters for the previously kicked-out stations) will be compared with $\sigma$. If the absolute value of residual of a previously kickedout station is now less than $\sigma$, then this station will become a kept one and will be used to estimate parameters in the next circle. As PCFC is concerned, FORT_ORD is kicked out in Circle 1, but it becomes a kept station in Circle 2, therefore the number of kicked-out stations is minus one. As shown in Table 5 , in the last two circles the estimations to parameters are almost the same. In addition, because of the increase in stations the error budget of parameters and the variance of residuals are decreased from Circle 2 to Circle 3. 
Table 6. The statistically selected on-plate stations on PCFC.

\begin{tabular}{|c|c|c|c|c|c|c|c|c|c|}
\hline \multirow[b]{2}{*}{ No } & \multirow[b]{2}{*}{ Sites } & \multirow{2}{*}{$\begin{array}{l}\text { Long. } \\
\text { (deg.) }\end{array}$} & \multirow{2}{*}{$\begin{array}{r}\text { Lat. } \\
\text { (deg.) }\end{array}$} & \multicolumn{3}{|c|}{ Uncertainty $(\mathrm{mm} / \mathrm{yr})$} & \multicolumn{3}{|c|}{ Residual (mm/yr) } \\
\hline & & & & $v_{x}$ & $v_{y}$ & $v_{z}$ & $v_{x}$ & $v_{y}$ & $v_{z}$ \\
\hline 1 & VNDNBERG & 239.38 & 34.56 & 0.527 & 0.584 & 0.614 & -0.022 & -0.045 & -0.056 \\
\hline 2 & FORT_ORD & 238.23 & 36.67 & 3.349 & 3.618 & 3.758 & 0.621 & -0.910 & -0.302 \\
\hline 3 & KAUAI & 200.33 & 22.13 & 0.324 & 0.279 & 0.330 & -0.211 & -0.518 & -0.850 \\
\hline 4 & KOKEE & 200.33 & 22.13 & 0.205 & 0.206 & 0.244 & 0.024 & 0.295 & 0.385 \\
\hline 5 & MK-VLBA & 204.54 & 19.80 & 0.624 & 0.573 & 0.573 & 0.567 & -0.022 & 0.487 \\
\hline
\end{tabular}

\section{Relative motions of VLBI stations on PCFC}

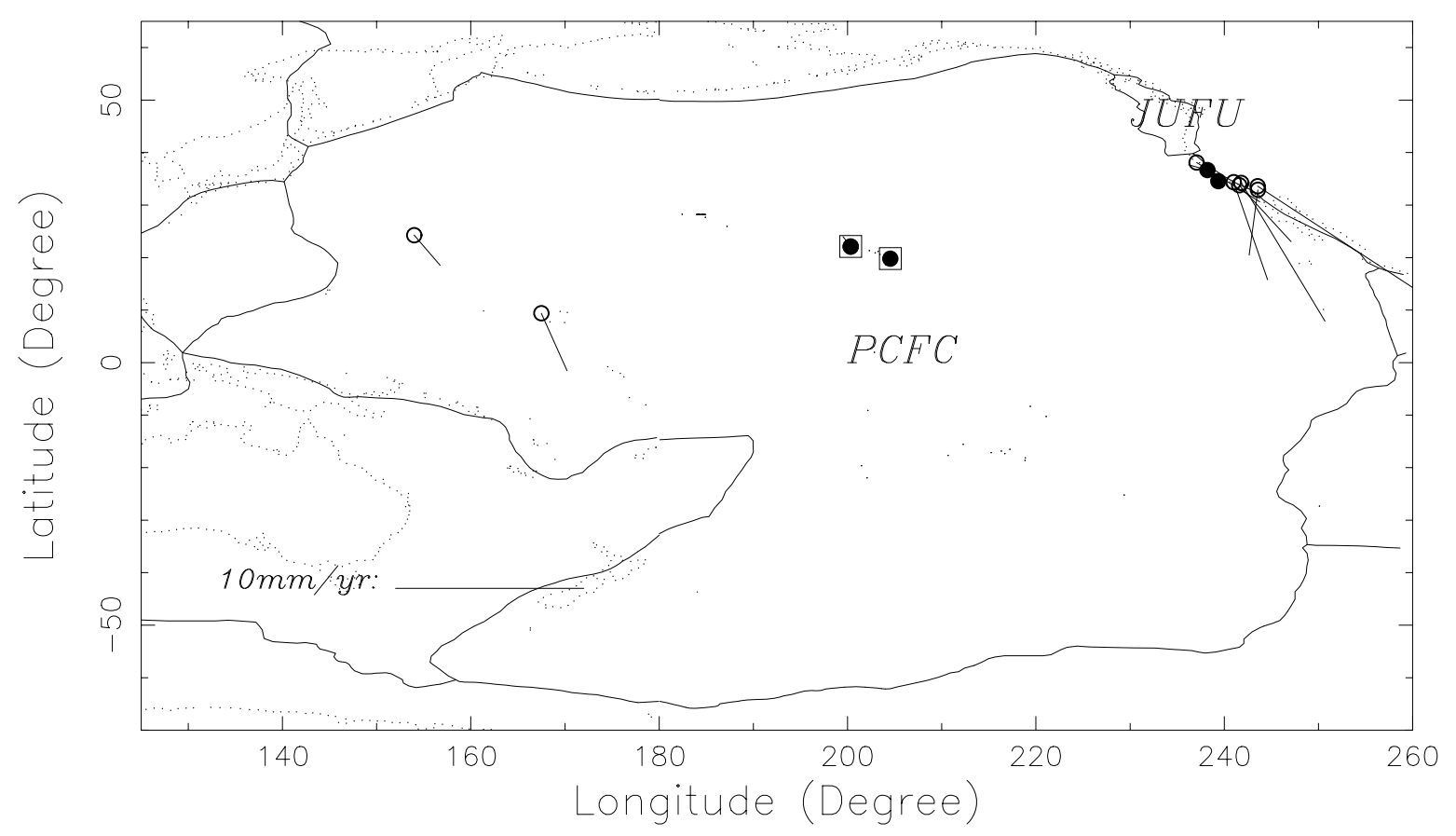

Fig. 3. The VLBI stations on PCFC (Symbols mean the same as in Fig. 1).

The statistically selected stations are shown in Table 6 and the geometric distribution of these stations is shown in Fig. 3. The symbols mean the same as in Fig. 1. As shown in Fig. 3, VNDNBERG and FORT_ORD are not VLBI primaries in ITRF2000 but are statistically selected as on-plate. By referring to the web page of Goddard Space Flight Center (GSFC VLBI Group, 1999) it is shown that the difference between the VLBI measurement of the motion of these two stations and the prediction of the plate motion model is similar to that of KOKEE and MK_VLBA in direction and magnitude, which testifies to the feasibility of the statistical selection method.

\subsection{The rest plates or blocks}

To estimate the seven systematic parameters in Eq. (7) by a general purpose least squares adjustment requires at least three observation points (corresponding to nine equations). On all the plates or blocks except NOAM, EURA and PCFC, the number of VLBI observation points is rather small. For instance, on Africa, Philippine, Australia, South America, Antarctica and Caribbean (AFRC, PHIL, AUST, SOAM,
ANTA and CARB), the number of points is respectively 2 , 2, 4, 3, 1 and 1, and the points with sufficient observations to determine the velocity are correspondingly 2, 0, 3, 2, 1 and 1. As far AUST is concerned, Eq. (7) can be directly applied to estimate the parameters, but the estimating and kickingout re-processing calculation cannot be applied. Therefore the statistical selection cannot be directly used but requires some special consideration. By applying Eq. (7) to AUST, some quantities of the three stations are listed in Table 7, which shows that the motion of PARKES is determined with the lowest precision and the corresponding absolute values of residuals are the largest among the three stations. If the variance is still checked with such a small sample, it is 0.07 . According to the $1 \sigma$ criterion PARKES should be kicked out. DSS45 and HOBART26 are primaries in ITRF2000, which is in consistency with the discussions here.

\subsection{A global selection}

For a global selection of on-plate sites, if the prediction of a plate motion model is directly used as the a priori value and applied Eq. (7) to all the observation points, then if the 
Table 7. The selection of on-plate sites on AUST.

\begin{tabular}{|c|c|c|c|c|c|c|c|c|c|}
\hline \multirow[b]{2}{*}{ No } & \multirow[b]{2}{*}{ Sites } & \multirow{2}{*}{$\begin{array}{l}\text { Long. } \\
\text { (deg.) }\end{array}$} & \multirow{2}{*}{$\begin{array}{r}\text { Lat. } \\
\text { (deg.) }\end{array}$} & \multicolumn{3}{|c|}{ Uncertainty $(\mathrm{mm} / \mathrm{yr})$} & \multicolumn{3}{|c|}{ Residual (mm/yr) } \\
\hline & & & & $v_{x}$ & $v_{y}$ & $v_{z}$ & $v_{x}$ & $v_{y}$ & $v_{z}$ \\
\hline 1 & DSS45 & 148.98 & -35.40 & 0.309 & 0.285 & 0.319 & -0.000 & -0.002 & -0.002 \\
\hline 2 & HOBART26 & 147.44 & -42.80 & 0.312 & 0.283 & 0.331 & -0.000 & 0.000 & 0.001 \\
\hline 3 & PARKES & 148.26 & -33.00 & 8.977 & 8.653 & 9.054 & 0.458 & 1.306 & 0.983 \\
\hline
\end{tabular}

Table 8 . The statistically selected on-plate sites.

\begin{tabular}{llllllll}
\hline \multicolumn{1}{c}{ Station } & Plate & \multicolumn{1}{c}{ Station } & Plate & \multicolumn{1}{c}{ Station } & Plate & Station & Plate \\
\hline DSS45 & AUST & ALGOPARK & NOAM & MARPOINT & NOAM & YUMA & NOAM \\
HOBART26 & AUST & FD-VLBA & NOAM & NL-VLBA & NOAM & FORT ORD & PCFC \\
DSS65 & EURA & FLAGSTAF & NOAM & NRAO_140 & NOAM & KAUAI & PCFC \\
EFLSBERG & EURA & GORF7102 & NOAM & NRAO20 & NOAM & KOKEE & PCFC \\
NYALES20 & EURA & HAYSTACK & NOAM & NRAO85 3 & NOAM & MK-VLBA & PCFC \\
ONSALA60 & EURA & HN-VLBA & NOAM & PIETOWN & NOAM & VNDNBERG & PCFC \\
WETTZELL & EURA & KP-VLBA & NOAM & RICHMOND & NOAM & FORTLEZA & SOAM \\
YEBES & EURA & LA-VLBA & NOAM & WESTFORD & NOAM & & \\
\hline
\end{tabular}

\section{Relative motions of VLBI stations}

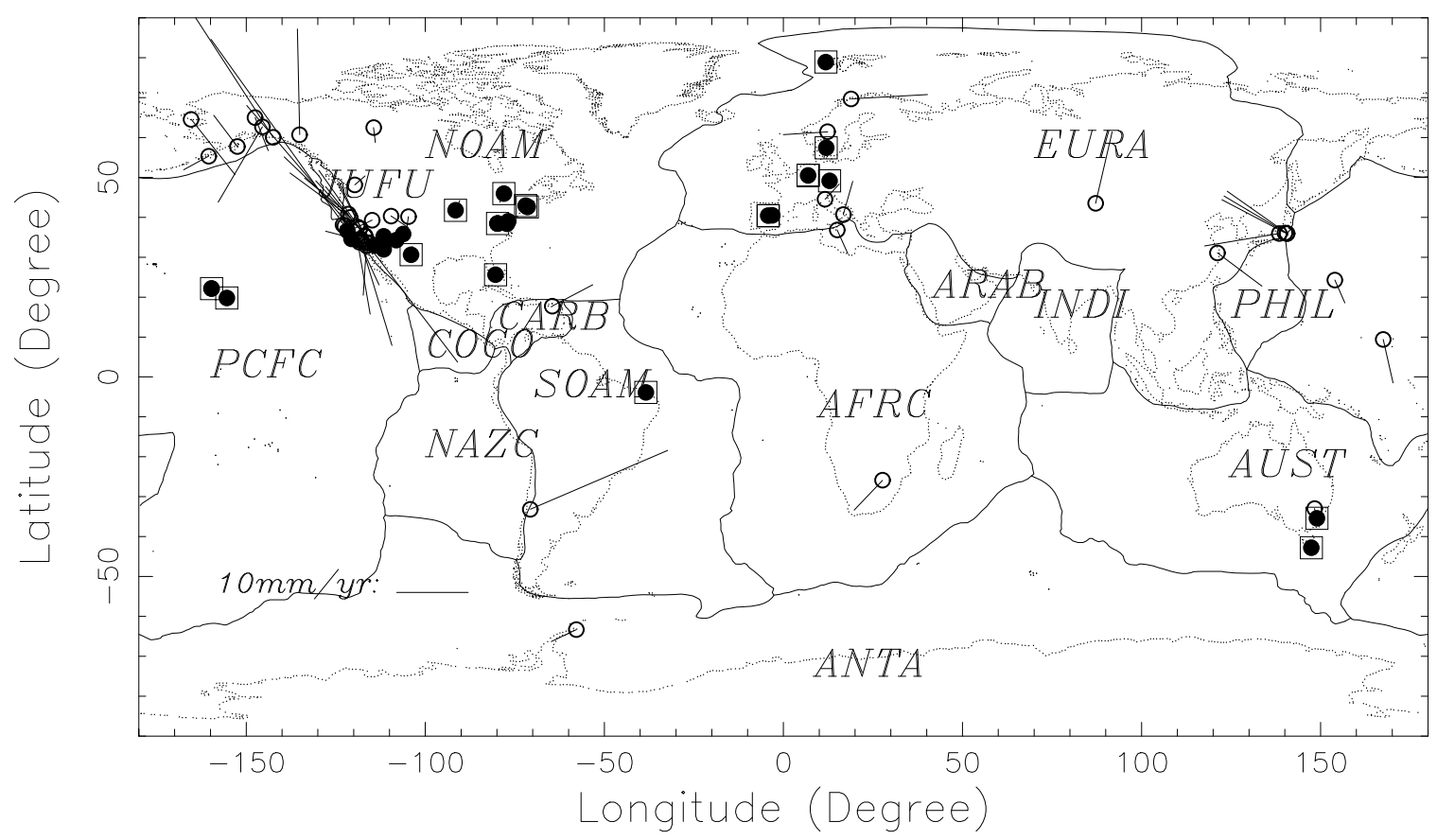

Fig. 4. A global statistical selection of on-plate sites and the comparison with primaries of ITRF2000 (Symbols mean the same as in Fig. 1).

modern observation and the model prediction are very close to each other for all the plates and blocks, a reasonable result can be obtained. However, if there is significant difference between the observation and the prediction for some of the plates or blocks, the related observation points would be kicked out because of the systematic bias in residuals, which would be obviously undesirable. With these considerations we perform the global selection as the following: (1) estimating the systematic parameters between the observation and the prediction by model of plate motion according to Eq. (7) for every possible individual plate or block; (2) unifying the station velocities into the system of the plate motion model according to the estimated parameters and (3) performing a global selection by taking all the observation points as on a single plate. With the distribution of the VLBI stations, the systematic parameters can be estimated only 
for NOAM, EURA, PCFC and AUST. After the systematic unification of velocities the result of the global selection is shown in Fig. 4 with symbols same as in Fig. 1. Table 8 shows the names of the 31 statistically selected on-plate stations.

There are six circles in the global selection. From Circle 1 to Circle 6 the variance is respectively 4.09, 2.06, 1.30, $1.04,0.94$ and 0.95 , the number of kicked-out stations is 33 , $8,6,1,1$ and 0 . The kicked-out station in Circle 4 is RICHMOND and in Circle 5 is GORF7102. Because the value of variance is not changed obviously from Circle 4 to 6 , the two stations are also shown in Table 8 as on-plate. As mentioned above, RICHMOND can be taken as on-plate but is not very firmly "fixed" on-plate in the statistical meaning. Since the insufficient observation points on AFRC, SOAM, ANTA, CARB and PHIL, the systematic parameters cannot be individually estimated by Eq. (7) and so we cannot make any systematic unification of related velocities before the global selection. Only FORTLEZA on South America is selected as on-plate and it is also a primary in ITRF2000. It is selected because the difference between the VLBI measurement and the model prediction of its motion is very small.

\section{Discussion}

Based on the analysis of the site motions determined by modern space geodetic techniques, we proposed a statistical selection of on-plate sites for the compilation of the conventional terrestrial reference frame. By applying this statistical method on a VLBI global solution, the selected onplate sites are more than and in consistency globally with the VLBI primaries of the ITRF2000. The statistical selection plays emphasis on the consistency between the motion of sites and the rigid motion of plates, rather than being limited to the distance from the site to the plate boundaries or deforming zones.

Some of the statistically selected on-plate sites, for instance, YUMA and FLAGSTAF on NOAM, FORT_ORD and VNDNBERG on PCFC, are not primaries in ITRF2000. However, by including these sites the estimations of plate rigid motion are not changed significantly. Instead, the pre- cision of parameters is increased due to the improvement in site geometric distribution, so these sites are recommended to be in the list of candidates for on-plate.

The details of our analysis show that RICHMOND can be taken as on-plate, but it is not in the list of those most firmly on-plate in a statistical meaning. It is usually used as one of the sites for the evolution of terrestrial reference frame in VLBI global analysis. We recommend using ALGOPARK, FD-VLBA or NRAO_140 as substitutes.

The statistically selected on-plate sites can be taken as a general selection, which as shown above is feasible. The geophysical information should be of course taken into consideration in the final check.

Acknowledgments. This work is partly supported by the Chinese national projects (G1998040703, No. 970231003), Chinese National Natural Science Foundation Committee (No. 19833030), Chinese Academy of Sciences (No. KJ951-1-304), Science and Technology Foundation of Shanghai City (No. JC14012).

\section{References}

Boucher, C., Current intercomparisons between CTS's, in Reference Frames, edited by J. Kovalevsky, I. I. Mueller, and B. Kolaczek, 327 pp., Kluwer Academic Publishers, 1989.

DeMets, C., R. G. Gordon, D. F. Argus, and S. Stein, Effect of recent revisions to the geomagnetic reversal time scale on estimates of current plate motions, Geophys. Res. Lett., 21, 2191-2194, 1994.

GSFC VLBI Group, Data products available electronically at http: // lupus.gsfc.nasa.gov/global, 1999.

Heki, K., Horizontal and vertical crustal movements from three-dimensional very long baseline interferometry kinematic reference frame: Implication for the reversal timescale revision, J. Geophys. Res., 101, 31873198, 1996.

Kogan, M. G., G. M. Steblov, R. W. King, T. A. Herring, D. I. Frolov, S. G. Egorov, V. Y. Levin, A. Lerner-Lam, and A. Jones, Geodetic constraints on the rigidity and relative motion of Eurasia and North America, Geophys. Res. Lett., 27, 2041-2044, 2000.

$\mathrm{Li}$, J. and G. Wang, Global solution of VLBI observations and assessments, Earth Planets Space, 52, 731-733, 2000.

MacMillan, D. S. and C. Ma, VLBI measurements of Caribbean and South American motion, Geophys. Res. Lett., 26, 919-922, 1999.

J. Li (e-mail: j11@center.shao.ac.cn), B. Zhang, M. Zhao, and G. Wang 